\title{
Increased preference for ethanol in rats following alcohol deprivation'
}

\author{
J. D. SINCLAIR AND R. J. SENTER \\ UNIVERSITY OF CINCINNATI
}

Depriving rats of alcohol, following a prolonged period of access to the solution, produced a much higher alcohol preference than did continual access. This "alcohol deprivation effect" is seen as possibly demonstrating a "need" for alcohol.

A major obstacle in studying alcoholism is the lack of a method which will produce in a laboratory animal a need for alcohol analogous to that shown by human alcoholics. Many experimental procedures have been used in an attempt to increase the preference for alcohol (e.g., Clay, 1964; Korman \& Stephens, 1960; Sirnes, 1953). Although factors have been found which do increase the preference, such increases have been relatively small and unreliable. No procedure has produced behavior indicating a persistent need for alcohol.

The writers have observed rats for whom a period of deprivation of alcohol (following a prolonged period of access to the solution) seems to produce a marked increase in alcohol preference once the solution is again made available. This observation has been made repeatedly, but under almost casual conditions. The present study was designed in an attempt to subject this observed "alcohol deprivation effect" to experimental test under controlled conditions.

Method

The Ss were 34 male hooded rats, approximately 5 months old, which had been used in an earlier study (Senter \& Sinclair, 1967) investigating the effects of thiamine deprivation on alcohol preference. ${ }^{2}$ During this earlier experiment the animals had 4 weeks of continual access to both $7 \%$ alcohol solution and water.

The rats were assigned to two equal groups, balanced for membership in the groups from the previous experiment. Group D was given access to Richter bottles containing a 7\% EtOH solution during Weeks 2, 4, 6, and 8 of the experiment only. Water was available ad lib at all times. Thus, Weeks $1,3,5$, and 7 constituted "alcohol deprivation periods." Group $C$ received continual access to alcohol and water for the duration of the experiment. There was no interruption of access to alcohol between the end of the previous study and the beginning of the present experiment.

Food was available to all Ss on an ad lib basis. The volume (in $\mathrm{ml}$ ) of EtOH solution and water consumed, and the weight of each rat, were recorded daily. The relative position of the EtOH and water bottles was alternated from cage to cage.

\section{Results}

The volume of EtOH solution divided by the total volume of liquid consumed daily by each rat $(\mathrm{E} / \mathrm{T})$ was used as a measure of preference for alcohol. The data from weeks following alcohol "deprivation" (1.e., 2, 4, 6, and 8) were combined. Thus, for Group $D$ the four $E / T s$ from the first days following the end of each alcohol deprivation period were summed and called Day 1, etc. The data of Group $C$ for these same weeks were treated the same way. Figure 1 shows the results for both groups. The high preference for alcohol immediately following alcohol deprivation (Day 1 ) is consistent over all rats in Group D, regardless of their treatment in the previous experiment. The subsequent decline in preference on the following six days is also consistent over Group D animals.

An analysis of covariance was used in an attempt to control for any differential effects from the previous experiment. The summed $\mathrm{E} / \mathrm{Ts}$ from the last week of the previous study were used as the covariate.

The between groups difference yielded an F-ratio

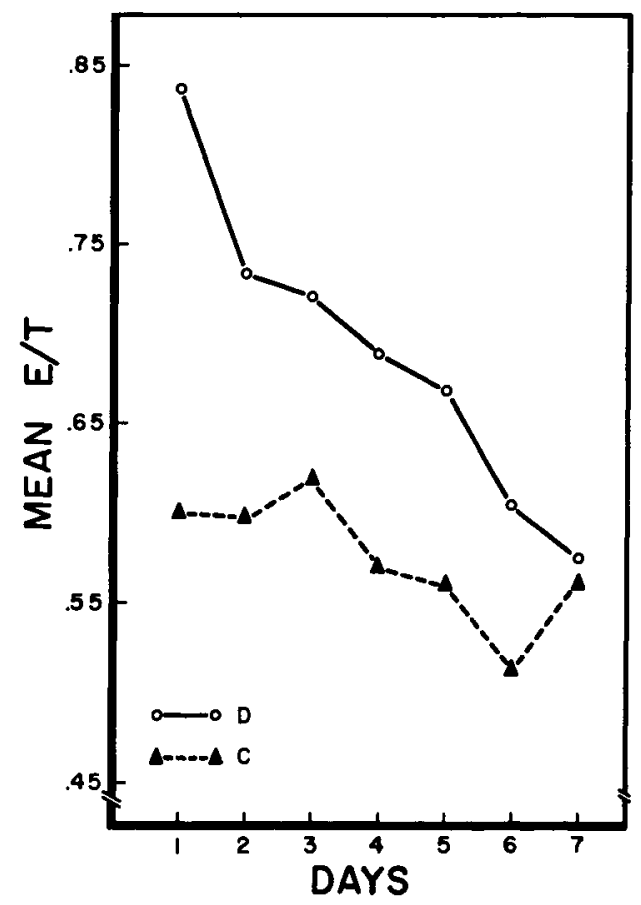

Fig. 1. Mean preference for EtoH for group $D$ during periods following "alcohol deprivation" and for group $C$ during the same time. For group D, "days" represent the number of days after the end of "alcohol deprivation." 
of $4.65(d f=1 / 30, p<.05)$. The $F$-ratio was elevated slightly by the analysis of covariance, but the significance level was unaltered $(F=6.82, d f=1 / 29, p$ $<.05)$. The F-ratios by days and groups by days interaction were significant beyond the .001 level $(F=24.11$, df $=6 / 359$ and $F=6.77$, df $=6 / 359$, respectively).

\section{Discussion}

The preference for alcohol increased greatly subsequent to each week of alcohol "deprivation." It is possible to see the behavior observed here as indicating the presence of a "need" for alcohol. Certainly animals with a need for a traditional commodity necessary to homeostatic balance (e.g., food or water) exhibit similar behavior patterns, specifically, initial ingestion of large amounts of the substance appropriate to their need, followed by a systematic decline in intake, approaching some "normal" base level. The question of how such a "need" could develop remains unanswered; observations of rats upon initial exposure to $\mathrm{EtOH}$ indicate that it is not developed in animals that have had no previous exposure to alcohol. It is felt that understanding this apparent "alcohol deprivation effect" in rats may give some clues to the development of the excessive need for alcohol shown by human alcoholics.

\section{References}

Clay, M. Conditions affecting voluntary alcohol consumption in rats. Quatt. J. Stud. Alc., 1964, 25, 36-55.

Korman, M., \& Stephens, H. Effects of training on the alcohol consummatory response in rats. Psychol. Rep., 1960, 6, 327-331.

Senter, R. J., \& Sinclair, J. D. Thiamine as an incentive in inducing alcohol consumption by rats. Submitted to Quart. J. Stud. Alc., 1967.

Simes, $\mathbf{T}$. Voluntary consumption of alcohol in rats with cirrhosis of the liver: a preliminary report. Quart. J. Stud. Alc., 1953, 14, 3-18.

\section{Notes}

1. This study represents a portion of the effort carried out under USPHS Grant No. MH 11895-01.

2. One animal was eliminated for placing food in his bottles, making data recording impossible. A second rat was eliminated because he showed a severe middle ear disease. 